\title{
SCIENTIFIC PROGRESS REPORT
}

During the past 12 month period the Valparaiso University group has been active on LAMPF experiment 969 , known as the MEGA experiment. This experiment is a search for the decay $\mu->$ e $\gamma$, a decay which would violate lepton number conservation and which is strictly forbidden by the standard model for electroweak interactions. Previous searches for this decay mode have set limit the present day limit of $4.9 \times 10^{-11}$. The MEGA experiment is designed to test the standard model predictions to one part in $10^{+13}$.

Progress on the experiment has been marked by research and development efforts applied to the complex detector system. Full scale segments of all detector components were assembled and placed in the superconducting solenoidal magnet in the stcpped muon channel at LAMPF during run period in 1989. Data collected from this run were helpful in evaluating detector performance, including the cylindrical wire chambers with associated electronics, the Fastbus-based data acquisition system, and the analysis software. Several redesigns were implemented in the post-run period as detector development and construction progressed. The fully configured detector will include seven smaller (dwarf) MWPCs, one larger, coaxial MWPC, and two scintillator barrels in the positron detector. The photon detector consists of four layers, each with an MWPC, a PDC, and a scintillator plane. Plans for the runs at LAMPF during 1990 will include three dwarf MWPCs, the larger coaxial MWPC, and both scintillator barrels in the positron detector and one fully configured layer in the photon detector.

Valparaiso University was responsible for the analysis of the data collected witl. the positron arm of the detector after the 1989 running period. In addition, we developed software for an off-Iine analysis of those events which survived our on-line software filter and were therefore event candidates 
for $\mu \rightarrow$ e $\gamma$. These programs were tested with Monte Carlo generated events both to confirm their ability to detect signal $\mu \rightarrow$ ey in triggered readouts which contained an average of 10 Michel positrons, and then to analyze triggered readouts with no $\mu->$ er signal in the frame and confirm that the patterns of hits do not accidentally conform to a signal event. Refinements in the algorithms to process the MWPC hits using a more discriminating analysis while retaining a very high signal identfication percentage was the focus of these activities. While the present codes are remarkably successful in finding real events ( $(808)$ and likewise successful in rejecting background accidental (fake) events $(<18)$, this work continues toward increasing and decreasing these percentages, respectively.

New multichamber algorithms were written and tested to reconstruct helical tracks from Michel positrons at low beam rates for detector calibration purposes. Applying these codes to the data collected in the 1990 running period will allow us to compare our Monte Carlo design assumptions with the actual resolutions achieved.

Special codes were likewise written and tested to reconstruct straight positron tracks from two neutrino stopped muon decays with the solenoidal magnetic field off. These codes are especially helpful in determining MWPC efficiency and alignment in the detector system. The codes are written to interact with the photon arm as well, taking signals from the PDCs and the scintillators caused by the positron traversal of the photon detector with the magnetic field off. Thus, relative alignments between the positron MWPCs and the photon arm PDCs and scintillators can be achieved as well.

Two physicists from Valparaiso University spent over two months in the summer 1989 at LAMPF working on both hardware and software. One of us (DDK) spent eight months in residence at LAMPF on university sponsored research 
leave. During this eight month period, a considerable fraction of the time was spent on hardware development and testing, including work on the MWPCs and on $R \& D$ for the muon stopping target.

One Valparaiso University student worked full time at LAMPF on the MEGA experiment during summer 1989. Three Valparaiso University students are working on MEGA during summer 1990. Valparaiso University has taken the responsibility for the further $R \& D$ and the production of the muon stopping targets for the experiment.

\section{DISCLAIMER}

This report was prepared as an account of work sponsored by an agency of the United States Government. Neither the United States Government nor any agency thereof, nor any of their employees, makes any warranty, express or implied, or assumes any legal liability or responsibility for :he accuracy, completeness, or usefulness of any information, apparatus, product, or process disclosed, or represents that its use would not infringe privately owned rights. Reference herein to any specific commercial product, process, or service by trade name, trademark, manufacturer, or otherwise does not necessarily constitute or imply its endorsement, recommendation, or favoring by the United States Government or any agency thereof. The views and opinions of authors expressed herein do not necessarily state or reflect those of the United States Government or any agency thereof. 

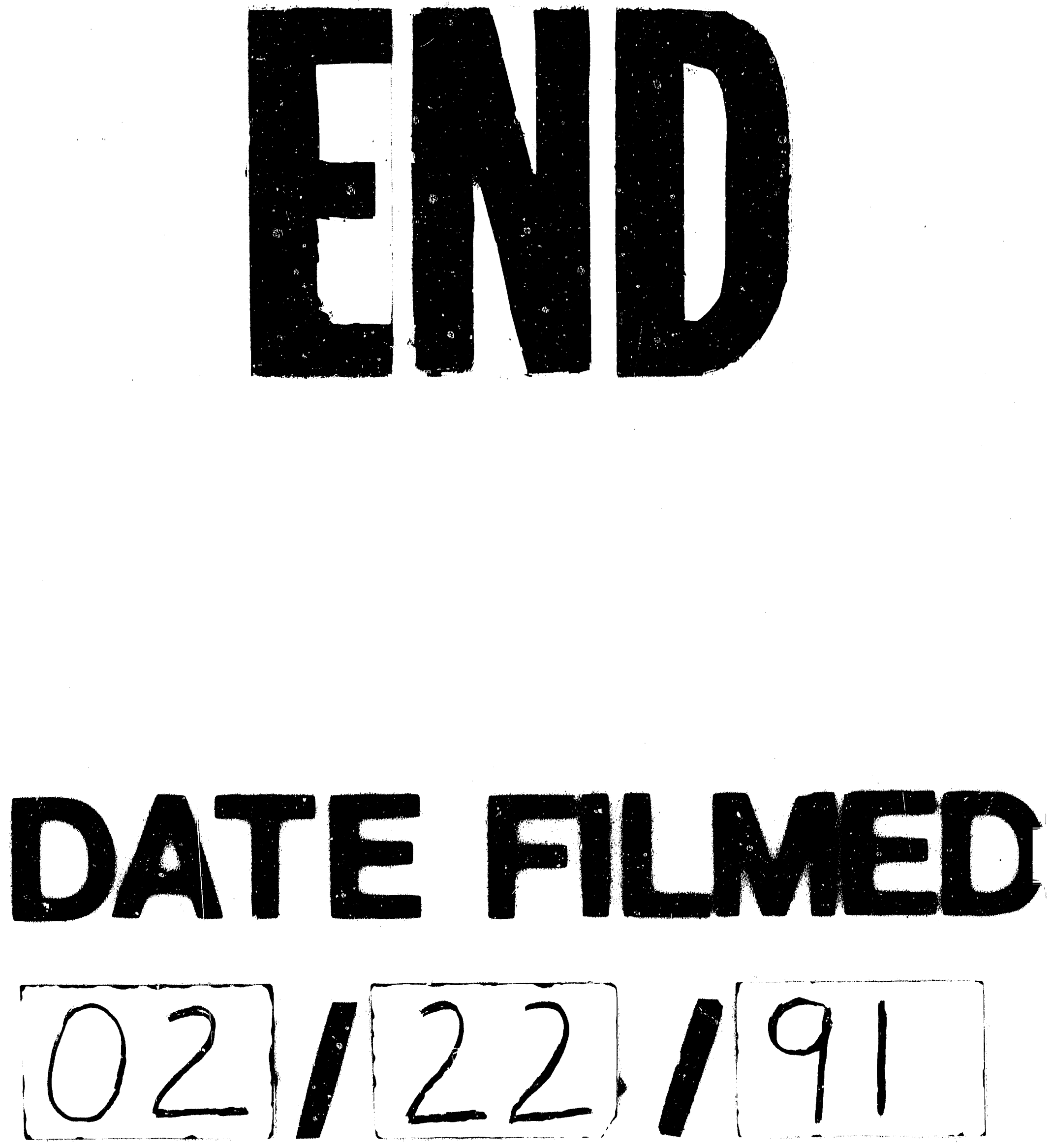
